\title{
An Adaptive Resolution Scheme for Performance Enhancement of a Web- based Multi-User VR Application
}

Rishabh Pathak, Anderson Augusto Simiscuka and Gabriel-Miro Muntean

IEEE BMSB 2021

IEEE BMSB 2021

Online
Presenter: Anderson Augusto Simiscuka, Dublin City University

$04 / 08 / 2021$

\section{(c) $(\mathrm{BY})$}

This project has received funding from the European Union's Horizon 2020 research and innovation programme under grant agreement No 870610 


\section{Introduction}

\section{Anderson Augusto}

\section{Simiscuka}

DCU
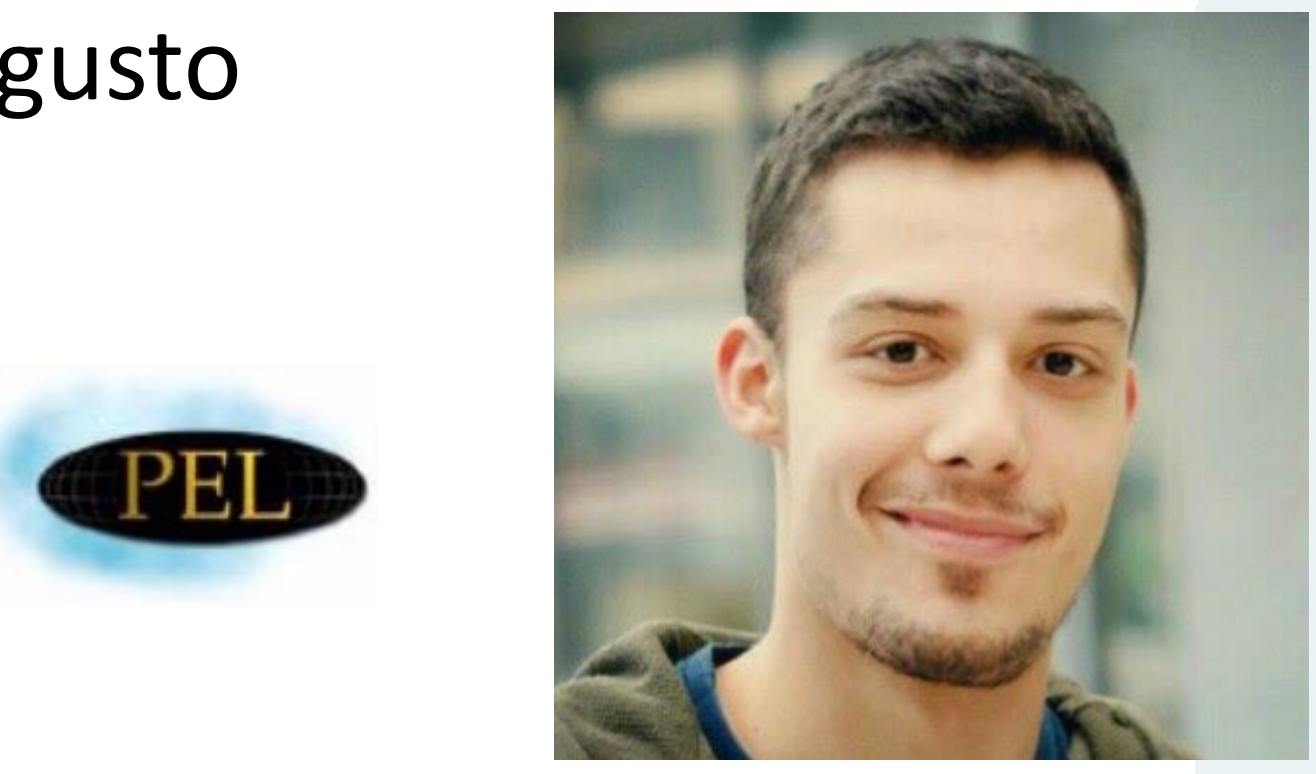

Doctor in Electronic Engineering

anderson.simiscuka2@mail.dcu.ie

\section{Postdoctoral Researcher}




\section{Motivation}

- The performance of web-based virtual reality (VR) applications, such as ones built with A-Frame, can be affected due to the limitations of the CPU and GPU, especially in multi-user applications.

- It is important to understand and analyze these applications' performance under different scenarios, demonstrating how an increase in the number of users affects metrics related to VR quality of experience (QoE).

- A smooth frame rate is important in VR applications to prevent motion sickness. 


\section{Multi-user VR Application}

- We propose a multi-user VR application developed using the A-Frame library, rendered as a $360^{\circ}$ experience within a browser.

- The application allows users to watch a film in a synchronized manner, in a virtual cinema room.

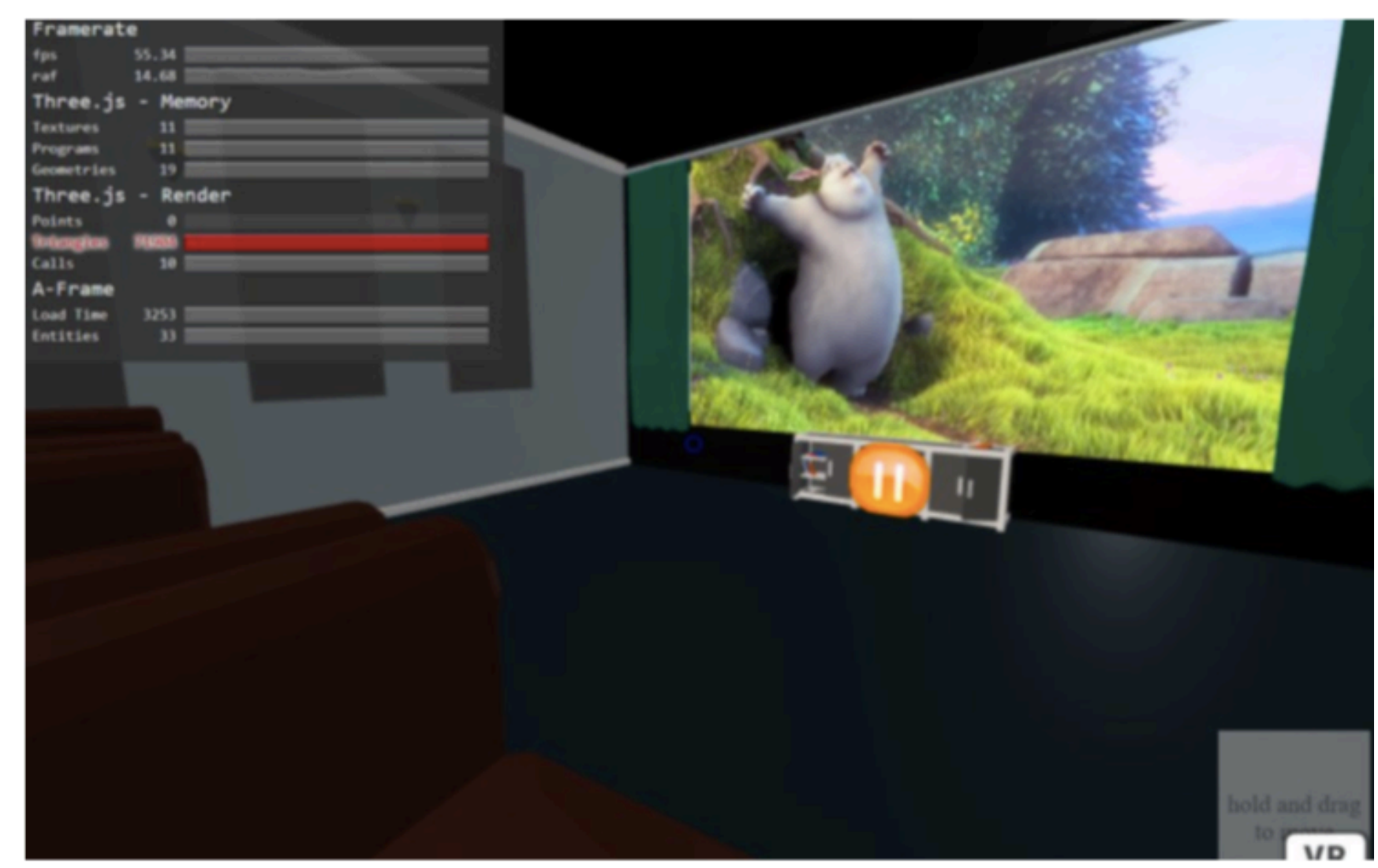




\section{Multi-user VR Application}

- The application employs a novel Adaptive Resolution Scheme for VR (ARS-VR), which improves VR applications' frame rate and frame latency on remote devices with limited processing and display.

- Users can move in the application, while WebRTC allows for voice chats. The library Networked A-Frame broadcasts synchronous playback to all clients.

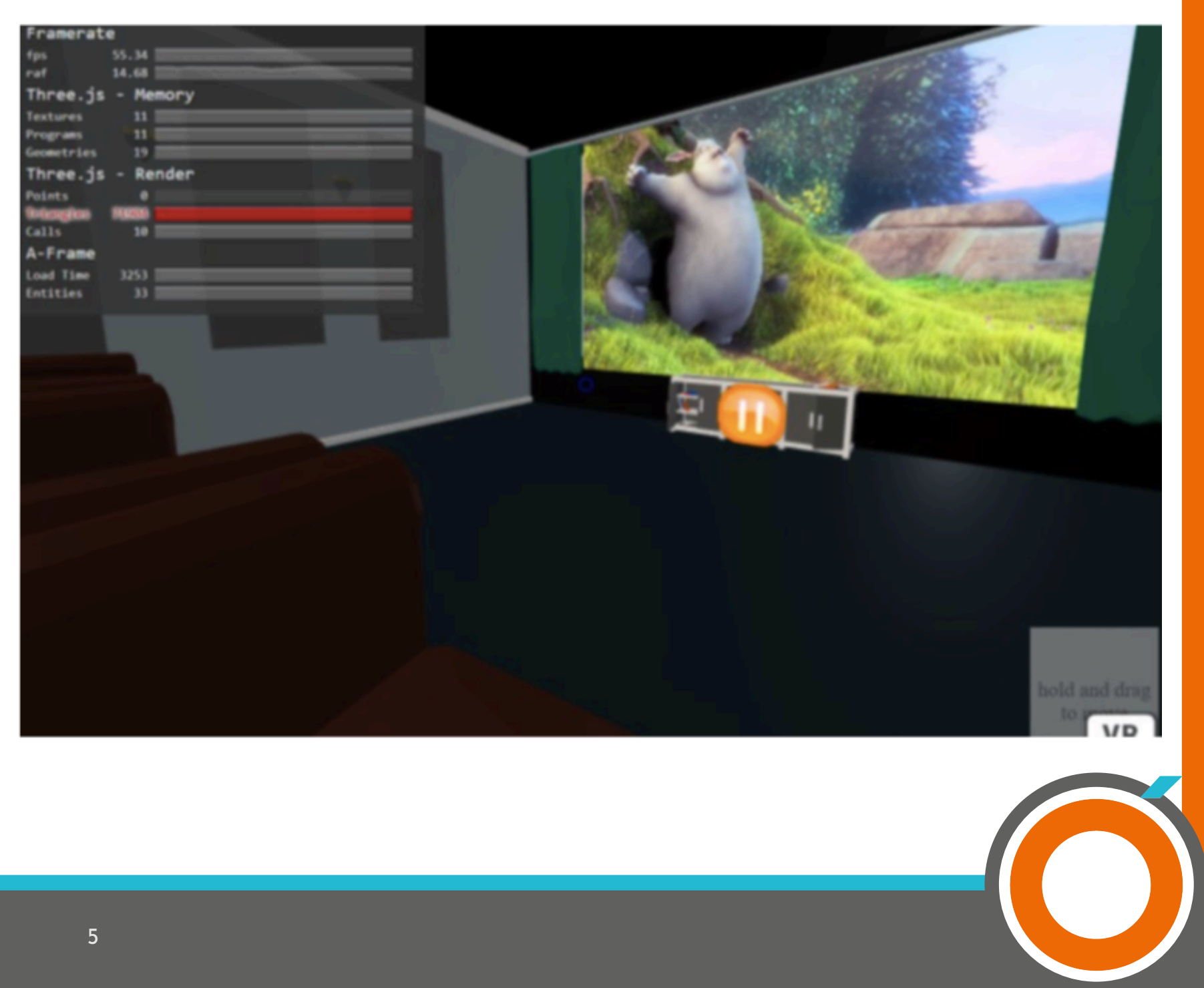




\section{VR Application Architecture}

- The application uses a reverse proxy service called ngrok to expose the local application server to a firewall on the public internet using secure tunnels.

- Users that are not on the same network as the server can access the application by querying the link to the secure tunnel.

- Two laptops were used as the application server and a client. A smartphone was used to simulate another client from a different network than the server.

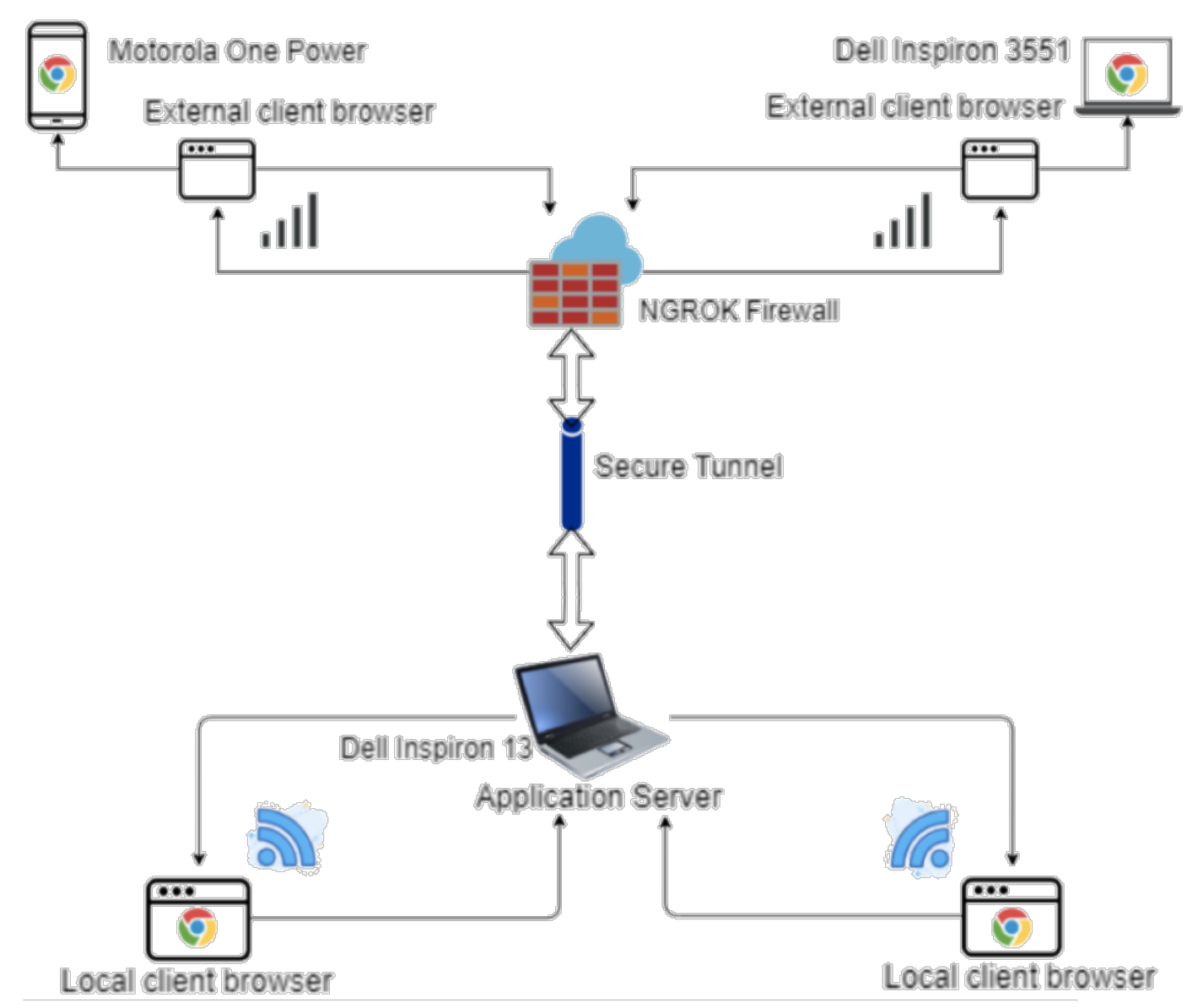




\section{Adaptive Resolution Scheme for VR}

- Once a connection is established, the server starts sending application data to the client and ARS-VR starts.

- The algorithm is deployed with JavaScript and it adjusts the resolution of the application by monitoring the frame rate.

- The numerator and denominator of the aspect ratio are multiplied with the frame rate to provide a dynamic resolution.

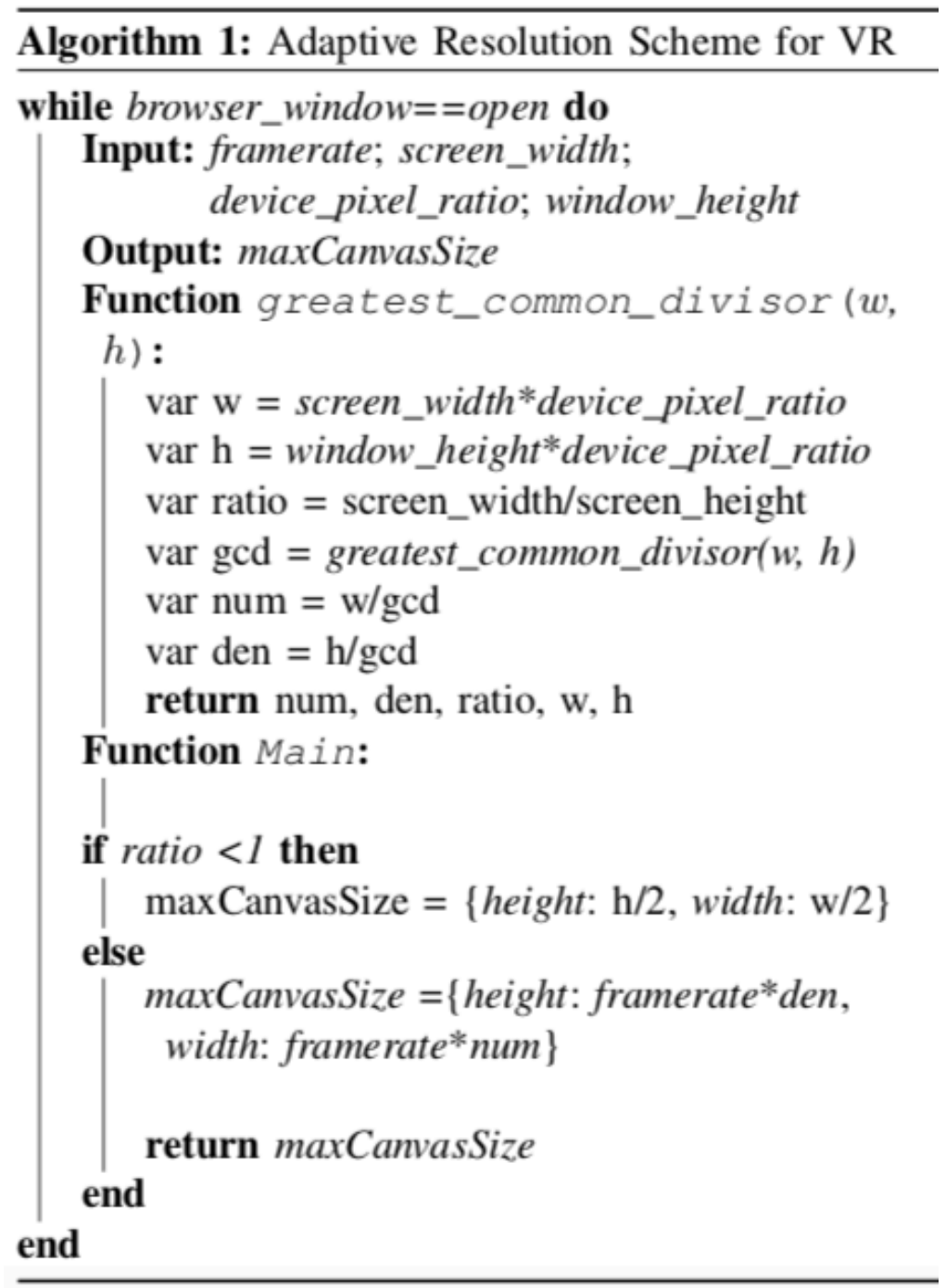




\section{Adaptive Resolution Scheme for VR}

- This is the main contribution of our work, as the VR application was built in order to test the approach.

- The approach can be applied to other web-based VR experiences as well.

- With the browser rendering the 3D scenarios in a lower resolution, the framerate gets closer to 60fps, which makes the VR experience more pleasant to users.

Algorithm 1: Adaptive Resolution Scheme for VR

while browser_window==open do

Input: framerate; screen_width;

device_pixel_ratio; window_height

Output: maxCanvasSize

Function greatest_common_divisor (w, h):

var $\mathrm{w}=$ screen_width $*$ device_pixel_ratio

var $\mathrm{h}=$ window_height $*$ device_pixel_ratio

var ratio $=$ screen_width/screen_height

var gcd = greatest_common_divisor $(w, h)$

var num $=\mathrm{w} / \mathrm{gcd}$

var den $=\mathrm{h} / \mathrm{gcd}$

return num, den, ratio, $\mathrm{w}, \mathrm{h}$

Function Main:

(1)

if ratio $<1$ then

maxCanvasSize $=\{$ height $: \mathrm{h} / 2$, width $: \mathrm{w} / 2\}$ else

maxCanvasSize $=\{$ height framerate $*$ den, width: framerate*num

return maxCanvasSize end 


\section{Performance Analysis}

- Browser used was Google Chrome.

- The frame rate was collected for 120 seconds from the application with the algorithm 'off' (i.e. using the default 1920px resolution) and then with the algorithm 'on' (i.e. with dynamic resolution).

- Scenarios with 4, 6 and 8 simultaneous users were considered. 


\section{Performance Analysis - FPS}

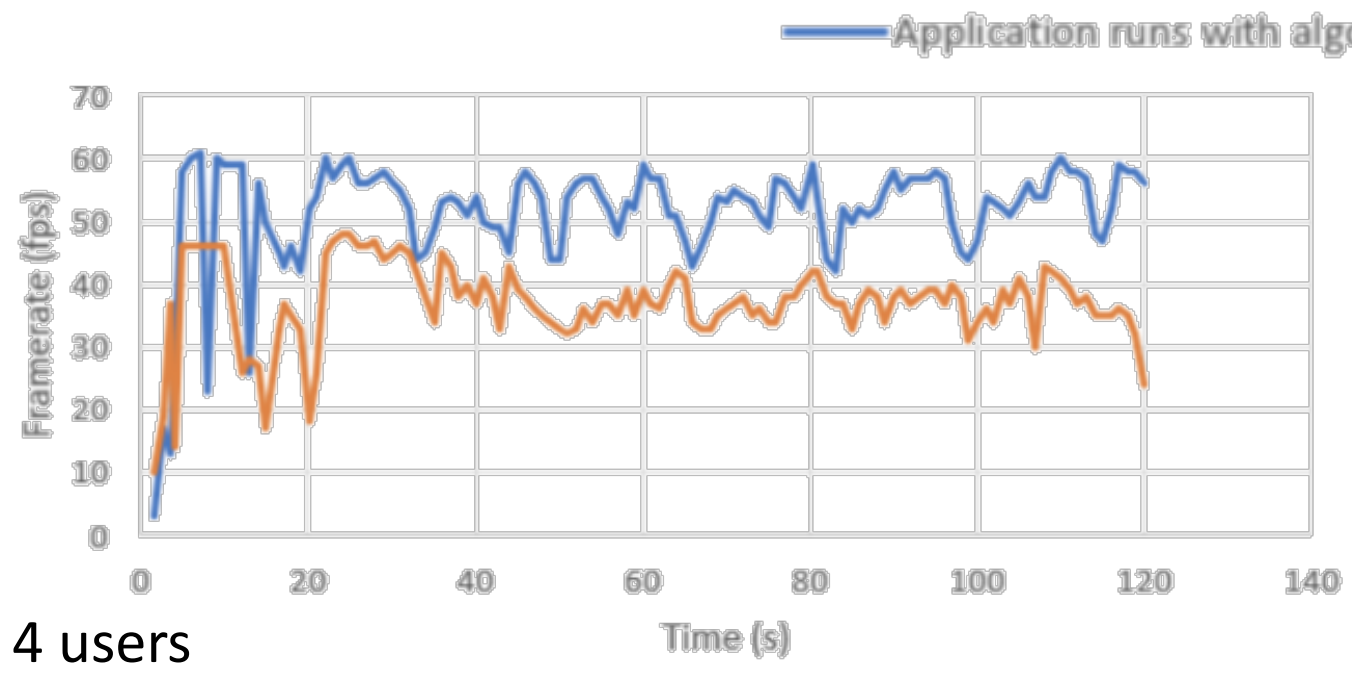

Application runs without algorithm
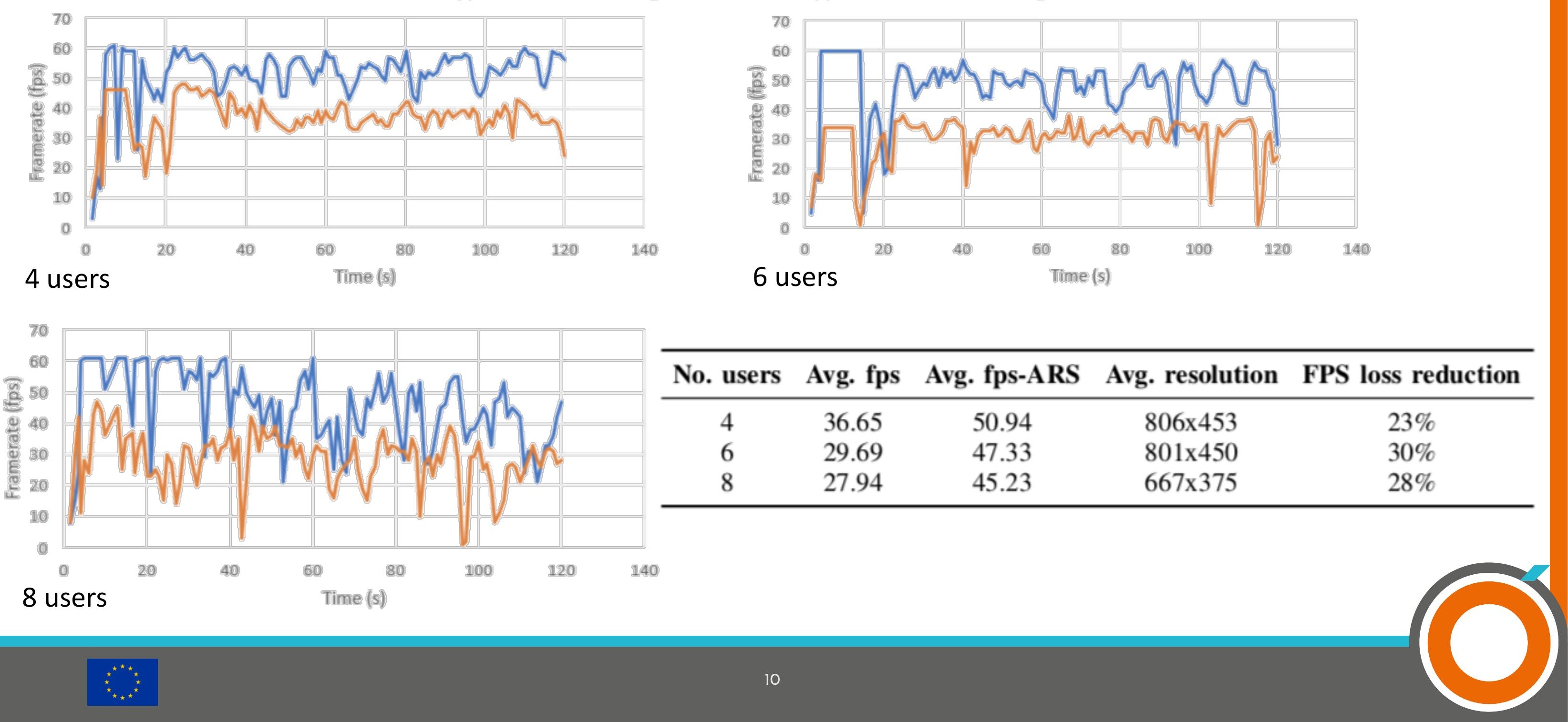


\section{Performance Analysis - Resolution}
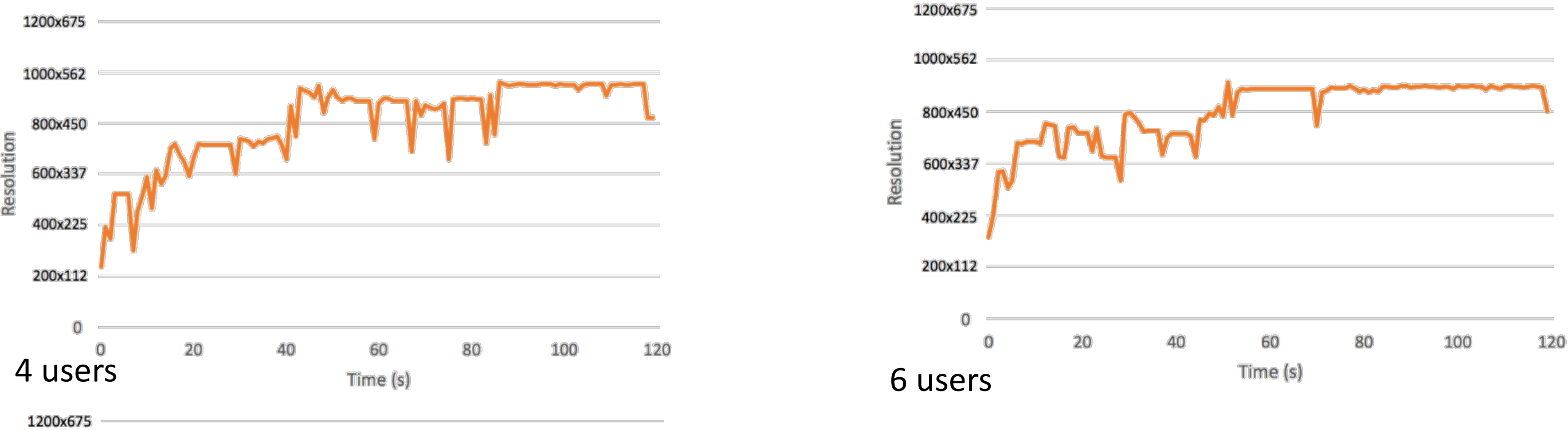

$1200 \times 675$

$1000 \times 562$

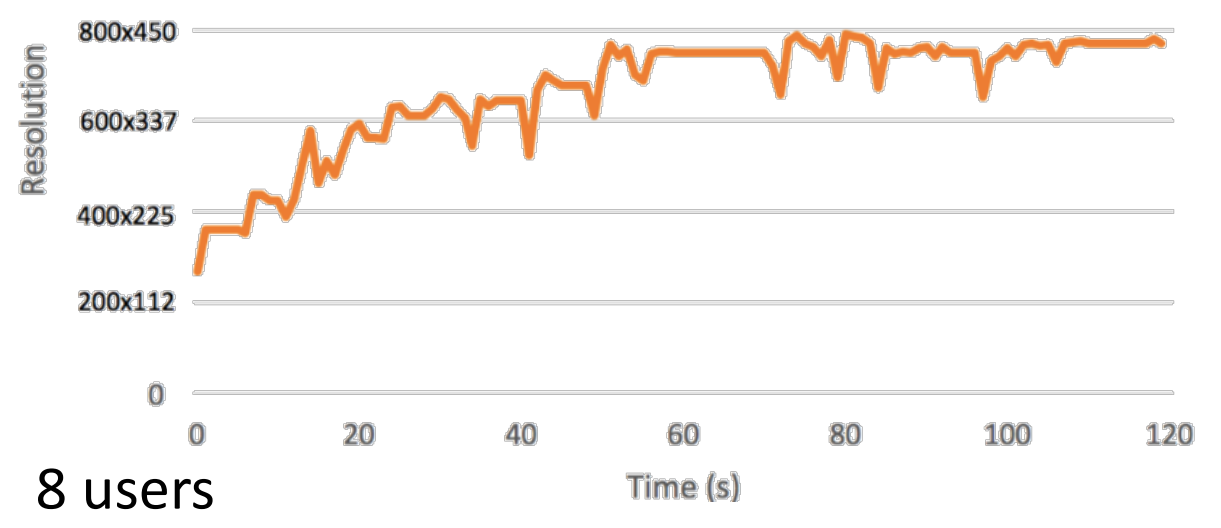

The resolutions, are on average $806 \times 453 p x$, $801 \times 450 p x$ and $667 \times 375 p x$ for the scenarios with 4, 6 and 8 users, respectively. 


\section{Performance Analysis - Frame Latency}
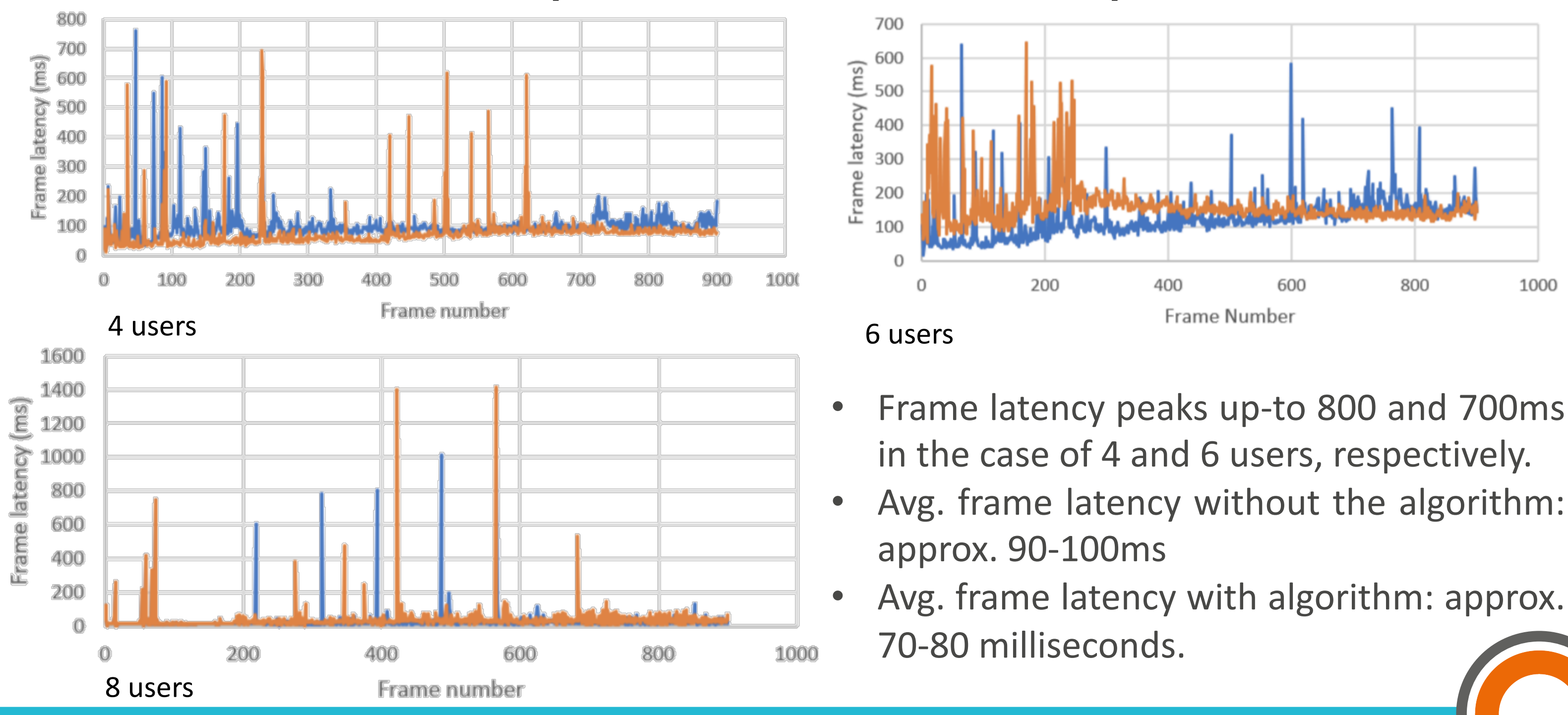

6 users

- Frame latency peaks up-to 800 and $700 \mathrm{~ms}$ in the case of 4 and 6 users, respectively.

- Avg. frame latency without the algorithm: approx. 90-100ms

- Avg. frame latency with algorithm: approx. 70-80 milliseconds. 


\section{Conclusion and Future Work}

- The A-Frame browser-based multi-user VR application can be rendered on remote devices with limited processing and display features.

- The ARS-VR algorithm was also introduced, aimed at improving VR performance in terms of frame rate and frame latency on such devices, with the dynamic adaptation of the visuals resolution.

- ARS-VR significantly reduces the FPS loss in the scenarios of 4,6 , and 8 simultaneous users by $23 \%, 30 \%$, and $28 \%$, respectively.

- Future work will improve the algorithm to support devices with different screen height and width ratios. Other user applications, such as video conferencing in A-Frame and other device types can also be studied. 


\title{
Thank you for your attention
}

\author{
Anderson Simiscuka, Dublin City University
}

(www.traction-project.eu

Anderson Simiscuka (DCU) anderson.simiscuka2@mail.dcu.ie www.eeng.dcu.ie/ pel 